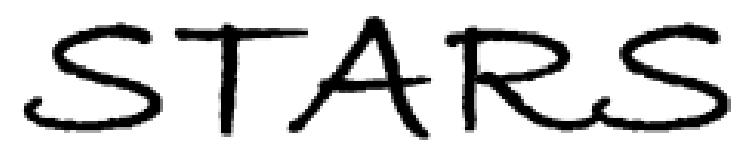

University of Central Florida

STARS

$1-1-2010$

\title{
Wave propagation in waveguide arrays with alternating positive and negative couplings
}

Nikolaos K. Efremidis

Peng Zhang

Zhigang Chen

Demetrios N. Christodoulides

University of Central Florida

Christian E. Rüter

See next page for additional authors

Find similar works at: https://stars.library.ucf.edu/facultybib2010

University of Central Florida Libraries http://library.ucf.edu

This Article is brought to you for free and open access by the Faculty Bibliography at STARS. It has been accepted for inclusion in Faculty Bibliography 2010 s by an authorized administrator of STARS. For more information, please contactSTARS@ucf.edu.

\section{Recommended Citation}

Efremidis, Nikolaos K.; Zhang, Peng; Chen, Zhigang; Christodoulides, Demetrios N.; Rüter, Christian E.; and Kip, Detlef, "Wave propagation in waveguide arrays with alternating positive and negative couplings" (2010). Faculty Bibliography 2010s. 133.

https://stars.library.ucf.edu/facultybib2010/133

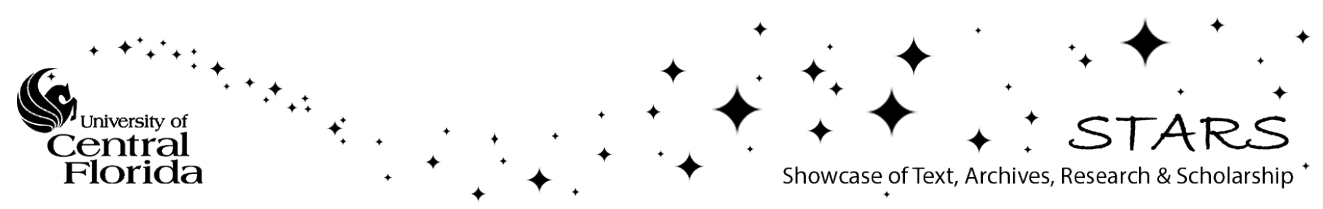


Authors

Nikolaos K. Efremidis, Peng Zhang, Zhigang Chen, Demetrios N. Christodoulides, Christian E. Rüter, and Detlef Kip 


\title{
Wave propagation in waveguide arrays with alternating positive and negative couplings
}

\author{
Nikolaos K. Efremidis, ${ }^{1}$ Peng Zhang, ${ }^{2}$ Zhigang Chen, ${ }^{2}$ Demetrios N. Christodoulides, ${ }^{3}$ Christian E. Rüter, ${ }^{4}$ and Detlef Kip ${ }^{4}$ \\ ${ }^{1}$ Department of Applied Mathematics, University of Crete, GR-71409 Heraklion, Crete, Greece \\ ${ }^{2}$ Department of Physics and Astronomy, San Francisco State University, San Francisco, California 94132, USA \\ ${ }^{3}$ Center for Research and Education in Optics and Lasers, School of Optics, University of Central Florida, Orlando, Florida 32816, USA \\ ${ }^{4}$ Helmut Schmidt University, Department of Electrical Engineering, D-22043 Hamburg, Germany
}

(Received 19 January 2010; published 11 May 2010)

\begin{abstract}
We introduce a waveguide array model with alternating positive and negative couplings between adjacent waveguides. Two different settings where such a model can be realized are identified as arrays of defects in Bragg gratings and arrays with propagation constants that periodically vary along the propagation direction. We analyze the properties of wave propagation in such waveguide arrays and find several interesting properties that have no counterpart in the case of arrays with constant couplings. These include the beam self-splitting, self-induced Talbot oscillations, symmetric evolution of Bloch oscillations, and new families of lattice solitons.
\end{abstract}

DOI: 10.1103/PhysRevA.81.053817

PACS number(s): 42.65.Tg, 42.82.Et

\section{INTRODUCTION}

The study of linear and nonlinear phenomena in waveguide arrays has attracted a lot of attention over the past decade. Such structures are associated with novel physical phenomena that arise from the interplay between periodicity and nonlinearity as well as with potential switching applications (see, for example, Ref. [1] for a recent review). In the linear regime, such waveguide settings have been studied ever since 1965 [2], but the field was pushed forward with new predictions and findings in the nonlinear regime, especially after the first prediction [3] and experimental demonstration [4] of discrete solitons. The transition from one-dimensional to two-dimensional structures [5] became possible later using the so-called optical induction technique [6]. Since then linear and nonlinear phenomena have been studied in a variety of discrete settings, including lattice solitons [7,8], discrete diffraction [9], Bloch oscillations [10-12], discrete Talbot patterns [13], Rabi oscillations $[14,15]$, and dynamic localization in curved waveguide arrays [16-19]. More complicated structures such as diatomic lattices or superlattices with alternating strong and weak couplings can lead to phenomena such as multiband diffraction relations [20-22], linear Shockley-type surface states [23], and nonlinear superlattice solitons [24]. Recently, three-dimensional lattices have been constructed using an optical induction technique [25].

The coupling in waveguide lattices is mathematically defined as a specific overlap integral between the linear modes of adjacent waveguides, and so far it has been positive in most of the works in this area. Negative coupling, however, has also been proposed [26,27] in the literature. In particular, it was shown [26] that negative coupling is possible in waveguide arrays supported by a Bragg structure [28]. Furthermore, the effective coupling can turn into zero in waveguides provided that the propagation constants periodically vary along the propagation direction in an out-of-phase fashion between adjacent waveguides [29-31]. The possibility of eliminating the coupling in double-well potentials was first analyzed in the context of quantum mechanics [32].

In this article we consider a waveguide lattice in which the magnitude of the coupling between successive waveguides is constant but the sign switches periodically between positive and negative values. We identify two different physical settings where such a configuration can be realized. We find several features of such arrays by analyzing their linear and nonlinear properties. In particular the diffraction dynamics lead to the splitting of the initial beam into two beams with opposite group velocities. Furthermore, Talbot patterns are spontaneously generated during propagation without the requirement of particular waveguide excitation (as in regular waveguide arrays). By introducing a linear variation in the propagation constant along the array, the system supports Bloch oscillations. We find that if the beam has an initial symmetric intensity profile, then the evolution leads to Bloch oscillations with a symmetric intensity pattern. Finally, the families of discrete solitons supported are analyzed by applying a transformation to the known solutions of the discrete nonlinear Schrödinger (DNLS) equation.

\section{PHYSICAL SETTINGS}

The mathematical model based on the coupled mode theory, which describes the light propagation in arrays with alternating coupling $(\mathrm{AC})$, is given by the following normalized AC-DNLS equation:

$$
i \frac{d u_{n}}{d z}+(-1)^{n} \kappa\left(u_{n-1}-u_{n+1}\right)+\gamma\left|u_{n}\right|^{2} u_{n}=0,
$$

where $u$ is the amplitude of the optical wave, $(-1)^{n} \kappa$ is the coupling coefficient between waveguides $n-1$ and $n, \gamma$ is the Kerr nonlinear coefficient, and $z$ is the propagation coordinate.

As so defined, the coupling coefficient switches between positive and negative signs periodically across the waveguide arrays. Negative coupling coefficients are physically equivalent to an additional phase shift of $\pi$ caused by the coupling. While this is not feasible for pure evanescent coupling in conventional waveguide arrays, we have identified two different physically realizable waveguide configurations where the coupling can have the form given by Eq. (1).

The first setting is related to waveguide arrays supported by a Bragg structure [26,28]. Let us briefly review how such a configuration can be used to realize negative coupling coefficients. We start from the paraxial wave equation

$$
i \psi_{z}+\frac{1}{2} \psi_{x x}+V(x) \psi+\gamma^{\prime}|\psi|^{2} \psi=0
$$


that describes the wave propagation in the presence of diffraction, Kerr nonlinearity, and a periodic potential $V(x)$. Since the mode of the $j$ th defect in isolation $\left[V_{j}(x)\right]$ satisfies the linear equation

$$
-q_{j} \chi_{j}+\frac{1}{2} \chi_{j, x x}+V_{j}(x) \chi_{j}=0,
$$

we can expand the solution of Eq. (2) as

$$
\psi(x, z)=e^{i q z} \sum_{j} \chi_{j}(x) u_{j}(z) .
$$

The resulting equation is of the DNLS type with a coupling coefficient between waveguides $n$ and $n-1$ equal to

$$
\kappa_{n}=\int_{-\infty}^{\infty}\left[V(x)-V_{n-1}(x)\right] \chi_{n}^{*}(x) \chi_{n-1}(x) d x,
$$

where $\int_{-\infty}^{\infty}\left|\chi_{j}(x)\right|^{2} d x=1$. In total-internal-reflection waveguide arrays the index contrast $V(x)-V_{n-1}(x)$, as well as the fundamental modes $\chi_{n}(x)$, are non-negative, and thus the coupling coefficient is always positive.

Now consider a Bragg structure with a low-index defect as shown in Fig. 1. The defect mode bifurcates from the edge of the first Brillouin zone into the first band gap, and for this reason its amplitude profile changes sign in adjacent high-index regions of the grating. In addition $V(x)-V_{n-1}(x)$ is less than or equal to zero. In [26] it is shown that the value of the coupling coefficient between two successive defects oscillates between positive and negative as the distance between the defects increases. Thus, positive and negative couplings can be made possible inside the same array by using two different spacings between the waveguides. Furthermore, it is possible to keep the spacing constant and use two different, appropriately engineered, Bragg structures to achieve the alternating coupling coefficients of Eq. (1).

The second realization of Eq. (1) consists of waveguides with propagation constants that vary periodically along the propagation direction [29-31]. In particular let us assume that the propagation constant of the $n$th waveguide varies as $\left(A_{n} / 2\right) \cos (\alpha z)$ and that the natural coupling coefficient

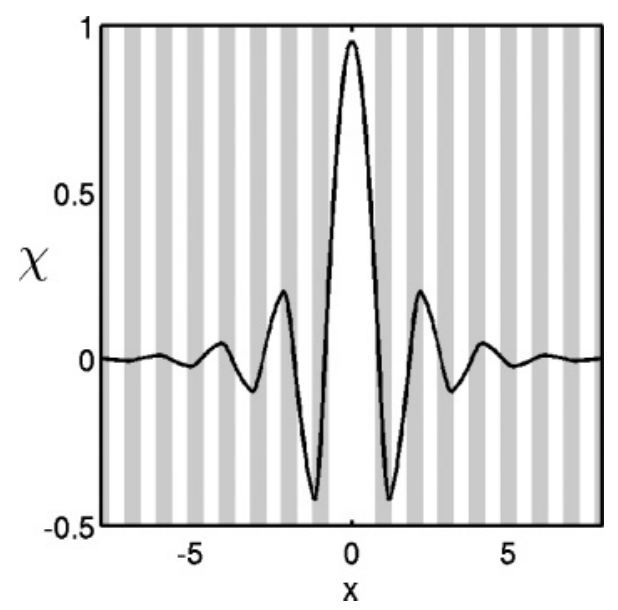

FIG. 1. Typical defect-mode field profile of a low-index defect supported by a Bragg structure. Gray regions correspond to higher refractive index. between successive waveguides is constant and equal to $\kappa_{0}$, that is,

$i \dot{\psi}_{n}+\kappa_{0}\left(\psi_{n-1}+\psi_{n+1}\right)+\frac{A_{n}}{2} \cos (\alpha z) \psi_{n}+\gamma\left|\psi_{n}\right|^{2} \psi_{n}=0$.

By applying the transformation

$$
\psi_{n}(z)=\phi_{n}(z) \exp \left(\frac{i A_{n}}{2 \alpha} \sin (\alpha z)\right),
$$

we obtain the following equation:

$$
i \dot{\phi}_{n}+\kappa_{n}^{\prime}(z) \phi_{n-1}+\kappa_{n+1}^{\prime}(z) \phi_{n+1}+\gamma\left|\phi_{n}\right|^{2} \phi_{n}=0
$$

which has a $z$-dependent coupling coefficient

$$
\kappa_{n}^{\prime}(z)=\kappa_{0} \exp \left(i \frac{\sin (\alpha z)}{2 \alpha}\left(A_{n-1}-A_{n}\right)\right) .
$$

This relation can be expanded using the Bessel generating function

$$
\begin{aligned}
\exp (i \sigma \sin \theta)= & J_{0}(\sigma)+2 \sum_{n>0}\left\{J_{2 n}(\sigma) \cos (2 n \theta)\right. \\
& \left.+J_{2 n-1}(\sigma) \cos [(2 n-1) \theta]\right\} .
\end{aligned}
$$

If the oscillations of the propagation constants are fast enough $(\alpha \gg 1)$ only the $z$-independent term in this expansion is significant and the effective coupling coefficient between waveguides $n-1$ and $n$ becomes

$$
\kappa_{n}=\kappa_{0} J_{0}\left(\frac{A_{n}-A_{n-1}}{2 \alpha}\right) \text {. }
$$

From Eq. (11) we see that the coupling becomes zero at the zeros of the Bessel function. Also there are particular ranges of parameters where the effective coupling is negative. In order to obtain the alternating signs in the coupling coefficients the amplitudes of the propagation constants of Eq. (6) are chosen to be periodic and to follow the pattern $[0,2 A, A,-A]$. Thus, the appropriate values of $\xi=A / \alpha$ can be determined from the equations $\kappa=\kappa_{0} J_{0}(\xi),-\kappa=\kappa_{0} J_{0}(2 \xi)$, or

$$
J_{0}(\xi)+J_{0}(2 \xi)=0 .
$$

The first few roots of the algebraic Eq. (12) are given by $\xi_{1} \approx 1.736, \xi_{2} \approx 5.739, \xi_{3} \approx 7.992, \xi_{4} \approx 12.016$.

We would like to point out that by applying the transformation

$$
u_{2 n}=v_{2 n}(-1)^{n}, \quad u_{2 n+1}=v_{2 n+1}(-1)^{n+1}
$$

to Eq. (1), we find that $v_{n}$ satisfies the DNLS equation. Thus there is a direct mapping between the DNLS equation and the AC-DNLS equation. However, the transformation (13) leads to initial conditions with complicated phase structures and for this reason, in most of the cases, it is preferable to analyze the properties of Eq. (1) from first principles.

\section{LINEAR PROPERTIES}

In the linear case $(\gamma=0)$ Eq. (1) supports solutions of the form

$$
u_{n}=\exp \left\{i\left[q n+\phi_{n}-E(q) z\right]\right\}
$$



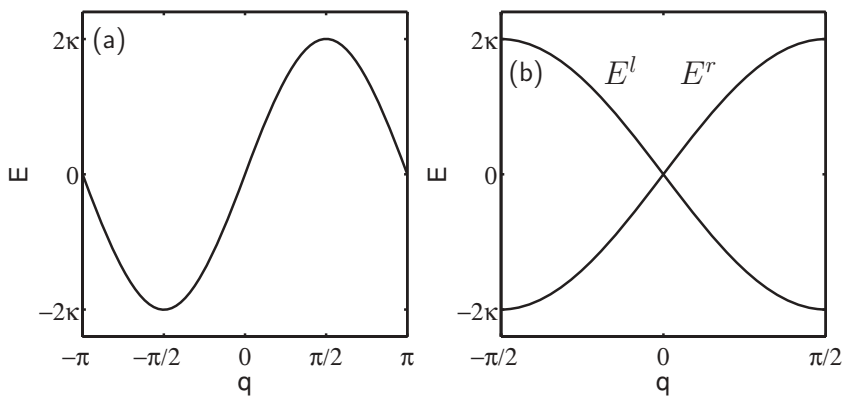

FIG. 2. Different forms of the diffraction curve of the AC-DNLS model as given (a) by Eq. (15) and (b) by Eq. (22).

where

$$
E(q)=2 \kappa \sin q
$$

is the sinusoidal diffraction curve shown in Fig. 2(a), $q$ lies inside the first Brillouin zone $-\pi \leqslant q \leqslant \pi$, and $\phi_{n}=$ $(\pi / 4)\left[1+(-1)^{n}\right]$. We would like to point out that, although the coupling is periodically alternating along the array, the diffraction relation does not have a gap which is a characteristic property of diatomic systems [20-22]. Thus Eq. (1) cannot be considered as a diatomic model. From Eq. (15) we can compute the group velocity

$$
v_{g}(q)=\frac{\partial E}{\partial q}=2 \kappa \cos q
$$

and the second-order diffraction coefficient

$$
d_{2}(q)=\frac{\partial^{2} E}{\partial q^{2}}=-2 \kappa \sin q .
$$

The general solution of Eq. (1) is given by

$$
u_{n}(z)=\int_{-\pi}^{\pi} C(q) e^{i\left[q n+\phi_{n}-E(q) z\right]} d q,
$$

where

$$
C(q)=\frac{1}{2 \pi} \sum_{n} e^{-i\left(q n+\phi_{n}\right)} u_{n}(0),
$$

and $u_{n}(0)$ is the initial condition. Notice that the continuouswave solutions (14) are chirped due to the presence of the phase term $\phi_{n}$. We would like to see which of these waves are excited when an initial unchirped continuous wave is launched in the waveguide array. In particular, by assuming a continuous wave which is incident at an angle $q_{0}$, that is,

$$
u_{n}(0)=\exp \left(i q_{0} n\right)
$$

we find that

$$
C(q)=\frac{e^{-i \pi / 2}}{2^{1 / 2}}\left[\delta\left(q_{0}-q\right) e^{i \pi / 4}+\delta\left(q_{0}-q-\pi\right) e^{-i \pi / 4}\right] .
$$

Thus a continuous wave of the form (20) is going to equally excite waves with Bloch momentum $q=q_{0}$ and $q=q_{0}+\pi$ with an additional phase difference between them. Note that these two waves are associated with opposite second-order diffraction coefficients $d_{2}=\mp \sin q_{0}$ and opposite group velocities $v_{g}= \pm 2 \kappa \cos q$. As a result an initial continuous wave with $q=q_{0}$ is going to excite one right-propagating wave and one left-propagating wave inside the lattice that has opposite diffraction coefficients. In particular, when $q_{0}=0$ the group velocities take their maximum values $\pm 2 \kappa$. By increasing the angle $q_{0}$ the group velocities decrease. Eventually for $q_{0}=$ $\pi / 2$, or in the middle of the Brillouin zone, both velocities become zero and the pulse does not split during propagation.

Although Eq. (15) is mathematically correct it does not provide direct physical intuition. For this reason we give an alternative definition of the diffraction curves [shown in Fig. 2(b)]

$$
E^{r}=2 \kappa \sin q, \quad E^{l}=-2 \kappa \sin q,
$$

which are associated with the right $u_{n}^{r}$ and left $u_{n}^{l}$ waves

$$
u_{n}^{r}=e^{i q n} e^{i \phi_{n}} e^{-i E^{r}(q) z}, \quad u_{n}^{l}=(-1)^{n} e^{i q n} e^{i \phi_{n}} e^{-i E^{l}(q) z},
$$

and the Brillouin zone is now defined in the region $-\pi / 2 \leqslant$ $q \leqslant \pi / 2$. This "physical" definition of the diffraction relations has the property that a continuous wave with a tilt $q_{0}$ equally excites the right $E^{r}\left(q_{0}\right)$ and the left $E^{l}\left(q_{0}\right)$ waves. In Fig. 2 both interpretations of the diffraction relation are shown.

The diffraction dynamics of the AC-DNLS equation can be obtained by solving Eqs. (18) and (19). In the case of a single waveguide excitation $u_{n}(0)=\delta_{n, n_{0}}$ the beam evolution is given by the following Green's function:

$$
G\left(n, n_{0} ; z\right)=J_{n-n_{0}}(2 \kappa z) e^{i \Phi_{n, n_{0}},}
$$

where $\Phi_{n, n_{0}}=\phi_{n}-\phi_{n_{0}}=(\pi / 4)\left[(-1)^{n}-(-1)^{n_{0}}\right]$. The diffraction dynamics of more complicated initial conditions $u_{n}(0)$ can be exactly found by using the formula $u_{n}(z)=\sum_{n^{\prime}} u_{n^{\prime}}(0) G\left(n, n^{\prime} ; z\right)$. However, in many cases the previous sum cannot be simplified.

Let us now consider a broad ( $M$ is large enough) Gaussian beam as the initial condition

$$
u_{n}(0)=e^{i q_{0} n} e^{-(n / 2 M)^{2}} .
$$

By following the relevant algebra it can be shown that asymptotically

$$
C(q) \approx \frac{\sqrt{2}}{2} e^{-i \pi / 2}\left[\delta_{M}\left(q_{0}-q\right) e^{i \pi / 4}+\delta_{M}\left(q_{0}-q \pm \pi\right) e^{-i \pi / 4}\right],
$$

where

$$
\delta_{M}(x)=\frac{M}{\sqrt{\pi}} e^{-(M x)^{2}} .
$$

Notice that, as $M$ increases, $\lim _{M \rightarrow \infty} \delta_{M}(x)=\delta(x)$ and this result becomes exact (rather than asymptotic) and identical to that of Eq. (21). From Eq. (26) we see that only a small part of the spectrum around $q=q_{0}$ and $q=q_{0}+\pi$ is excited. As a result, the diffraction relation can be approximated by keeping the first three terms of its Taylor expansion around $q_{1}=q_{0}$ and $q_{1}=q_{0}+\pi$, that is,

$$
E(q) \approx 2 \kappa \sin q_{1}+2 \kappa \cos q_{1}\left(q-q_{1}\right)-\kappa \sin q_{1}\left(q-q_{1}\right)^{2} .
$$

By utilizing Eq. (28) we find that

$$
u_{n}(z) \approx \frac{\sqrt{2}}{2} e^{-i \pi / 2} e^{i \phi_{n}}\left[e^{i \pi / 4} F\left(q_{0}\right)+e^{-i \pi / 4} F\left(q_{0}+\pi\right)\right],
$$



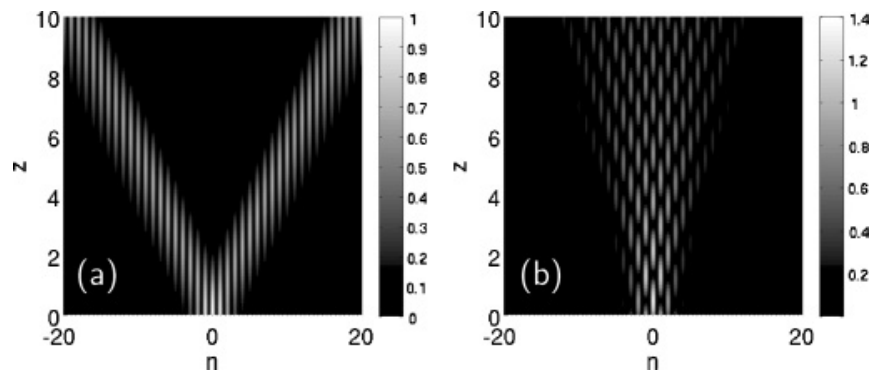

FIG. 3. Intensity diffraction patterns of a Gaussian beam with tilt (a) $q_{0}=0$ and (b) $q_{0}=\pi / 2$.

where

$$
\begin{aligned}
F\left(q_{0}\right)= & e^{i q_{0} n} e^{-2 i \kappa \sin \left(q_{0}\right) z}\left(\frac{M^{2}}{M^{2}-i \kappa \sin \left(q_{0}\right) z}\right)^{1 / 2} \\
& \times \exp \left(-\frac{1}{4} \frac{\left[n+2 \kappa \cos \left(q_{0}\right) z\right]^{2}}{M^{2}+i \kappa \sin \left(q_{0}\right) z}\right)
\end{aligned}
$$

Equations (29) and (30) are in agreement with the results obtained using the diffraction relation. In particular, we see that an initial beam splits into two waves propagating with opposite velocities $v_{g}= \pm 2 \kappa \cos q_{0}$ and diffraction coefficients $d_{2}=$ $\mp 2 \kappa \sin q$. In the case $q_{0}=0$ the group velocities take their maximum values $v_{g}= \pm 2 \kappa$ and both beams have zero secondorder diffraction. On the other hand, in the middle of the Brillouin zone, $q_{0}=\pi / 2$, both group velocities become zero and the magnitude of the second-order diffraction is maximum, $d_{2}=\mp 2 \kappa$ (half of the beam experiences negative and half of the beam experiences positive second-order diffraction). In Fig. 3 the diffraction pattern of an initial beam of the form $\exp \left[-(n / 3)^{2}\right] \exp \left(i q_{0} n\right)$ is shown for $q_{0}=0, \pi / 2$.

An interesting property of Fig. 3(b) is the Talbot patterns that are self-generated during propagation: Periodically, light switches between the even and the odd waveguides. Note that in regular arrays such Talbot oscillations are observed only when periodically selective waveguides are excited in the array [13]. Here the Talbot oscillations with a broad beam arise from the alternating positive and negative couplings. Such a pattern is more clearly shown in Fig. 4(a), where a broader Gaussian beam is used ( $M=5$ and $\left.q_{0}=\pi / 2\right)$ as the initial condition. To analyze the dynamics, we can employ Laplace's method for the asymptotic evaluation of the integral (18) which is valid in the limit of large $M$. However, the exact same result is obtained
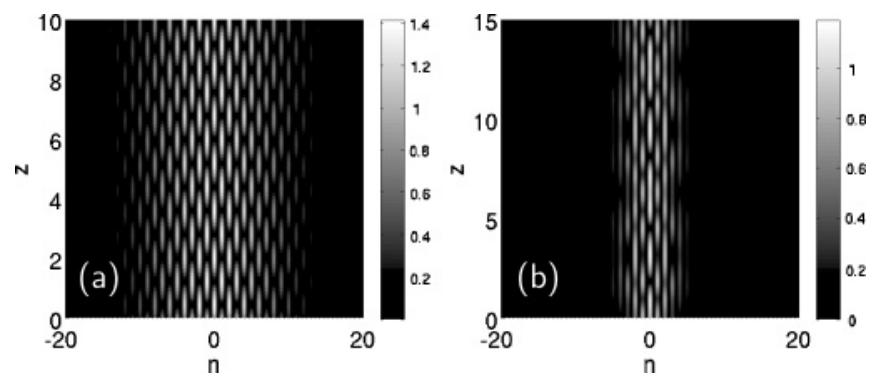

FIG. 4. (a) Talbot pattern generated by a broad Gaussian beam with a tilt $\pi / 2$ and (b) Bloch oscillations of a Gaussian beam with $M=3 / 2$ and $q_{0}=0$. simply by assuming a continuous wave as an initial condition. In particular, our calculations show that

$$
\begin{gathered}
u_{2 n}(z)=\sqrt{2} e^{i q_{0} 2 n} \cos \left(\frac{\pi}{4}-2 \kappa \sin \left(q_{0}\right) z\right), \\
u_{2 n+1}(z)=\sqrt{2} e^{i q_{0}(2 n+1)} \sin \left(\frac{\pi}{4}-2 \kappa \sin \left(q_{0}\right) z\right) .
\end{gathered}
$$

Equations (31) and (32) describe the oscillations depicted in Fig. 4(a). Note that the period of the intensity oscillations is given by $z_{0}=\pi /\left(2 \kappa \sin q_{0}\right)$. Thus, if $q_{0}=\pi / 2$ the period is minimum $z_{0}=\pi / 2 \kappa$. On the other hand, when $q_{0}=0$ the period becomes infinite and Talbot oscillations disappear.

By introducing an additional linear tilt in the propagation constant $\beta n u_{n}$ in Eq. (1), Bloch oscillations are expected to take place. In this case the Green's function for a single waveguide excitation is given by

$$
\begin{aligned}
G\left(n, n_{0} ; z\right)= & J_{n-n_{0}}\left[\frac{4 \kappa}{\beta} \sin \left(\frac{\beta z}{2}\right)\right] \\
& \times \exp \left(\frac{i\left(n-n_{0}\right)}{2} \beta z+i \Phi_{n, n_{0}}\right) .
\end{aligned}
$$

The normal modes of this system are the localized WannierStark modes $\psi_{n, n_{0}}=A_{n, n_{0}} \exp \left(-i E_{n_{0}} z\right)$ with

$$
A_{n, n_{0}}=J_{n_{0}-n}\left(\frac{2 \kappa}{\beta}\right) \exp \left(i \Phi_{n, n_{0}}+i \frac{\pi}{2}\left(n_{0}-n\right)\right)
$$

and $E_{n_{0}}=\beta n_{0} / \kappa$. We would like to point out that $A_{2 n_{0}-n, n_{0}}=$ $A_{n, n_{0}}$ (i.e., the mode $A_{n, n_{0}}$ is an even function of $n-n_{0}$ ). Notice that the motion of the center of the beam, $\langle x\rangle=$ $\sum_{n} n\left|u_{n}\right|^{2} / \sum_{n}\left|u_{n}\right|^{2}$, remains unchanged, $d\langle x\rangle / d z=0$, for initial profiles of the form $u_{n}=v_{n} \exp (i \phi n), v_{n}>0$, as opposed to lateral oscillation in the case of arrays with constant coupling.

\section{SOLITON SOLUTIONS}

In the nonlinear case, it is straightforward to find the characteristic amplitude profiles of the families of soliton solutions supported by Eq. (1). This can be done by utilizing Eq. (13) to transform the well-known solutions of the DNLS to the solutions of the AC-DNLS equation. We focus in the case of self-focusing nonlinearity $(\gamma=1)$, although these calculations can be carried out also in the case of self-defocusing nonlinearity. In particular, there are two fundamental families of soliton solutions of the self-focusing DNLS equation: (a) the on-site solution that has a maximum on a lattice site [3] and (b) the intrasite (or off-site) solution that attains its maximum between two successive lattice sites [33]. Both of these solutions are in phase (i.e., the phase difference between successive sites is zero). Note that if $v_{n}$ is a solution of the DNLS equation then $v_{n-n_{0}}$ is also a solution for arbitrary $n_{0}$.

The transformations given by Eqs. (13) introduce an additional phase difference of the form $0,0, \pi, \pi, 0,0, \pi$, $\pi, \ldots$, into successive elements of the DNLS solitons. As a result, if $u_{n}$ is a solution of the AC-DNLS then $u_{n-2 n_{0}}$ is also a solution, meaning that a single solution of the DNLS transforms into two different solutions of the AC-DNLS. However, only one of these solutions exists in each lattice site. In particular, the on-site solution transforms to the solutions shown in Figs. 5(a) and 5(b). According to the numbering 

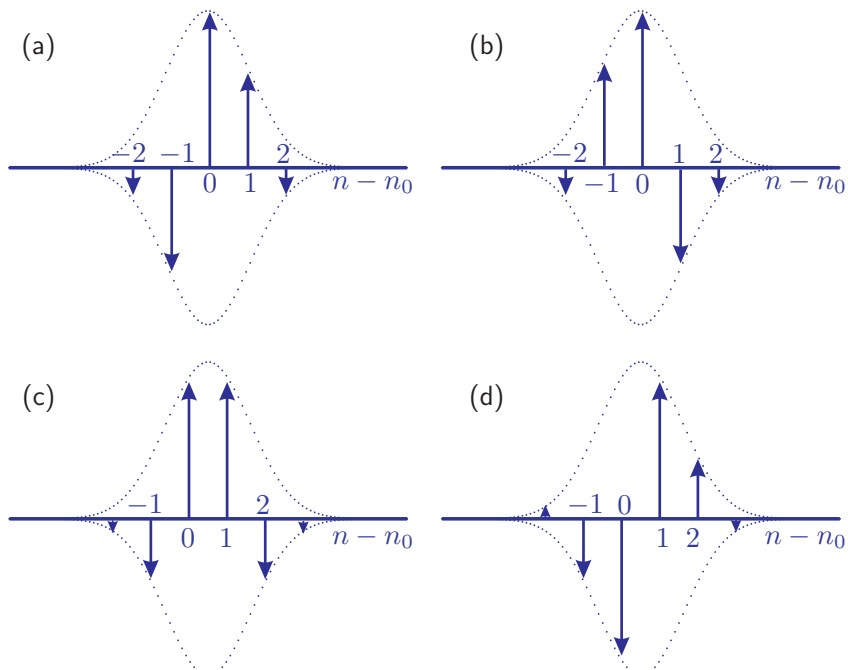

FIG. 5. (Color online) Sketch of typical amplitude profiles, each one belonging to a different family of soliton solutions, supported by the AC-DNLS equation as a function of $n-n_{0}$. According to the sign of the coupling between $n_{0}$ and $n_{0}+1$ either solutions (a) and (c) or solutions (b) and (d) are supported.

shown in this figure, mode (a) exists in the cases of positive coupling between waveguides $n_{0}$ and $n_{0}+1$ whereas mode (b) exists in the cases of negative coupling between waveguides $n_{0}$ and $n_{0}+1$. In a similar fashion the intrasite solution of the DNLS equation, which has maximum intensity between two successive waveguides, transforms to the solutions shown in Figs. 5(c) and 5(d). Furthermore, the power versus propagation constant curves as well as the stability of these solutions are identical to those of the DNLS equation. In particular, the on-site mode is stable whereas the intrasite mode is unstable due to oscillatory instabilities. The configurations presented in this section are experimentally realizable. However, in the case of relatively broad soliton solutions (occupying more than two lattice sites) proper phase engineering of the excitation beam might be required.

\section{CONCLUSIONS}

We have introduced and analyzed a model that accounts for alternating positive and negative couplings in waveguide lattices. We have identified two different settings where such a model can be applicable. Several interesting properties unique to these waveguide lattices were found, including self-splitting of an input beam into a right- and a left-propagating wave, self-induced Talbot patterns, and symmetric evolution of Bloch oscillations. In the nonlinear case, families of supported lattice solitons were analyzed by directly transforming solutions of the DNLS equation. Experimental investigation of these intriguing phenomena is currently underway.
[1] F. Lederer, G. I. Stegeman, D. N. Christodoulides, G. Assanto, M. Segev, and Y. Silberberg, Phys. Rep. 463, 1 (2008).

[2] A. L. Jones, J. Opt. Soc. Am. 55, 261 (1965).

[3] D. N. Christodoulides and R. I. Joseph, Opt. Lett. 13, 794 (1988).

[4] H. S. Eisenberg, Y. Silberberg, R. Morandotti, A. R. Boyd, and J. S. Aitchison, Phys. Rev. Lett. 81, 3383 (1998).

[5] J. W. Fleischer, M. Segev, N. K. Efremidis, and D. N. Christodoulides, Nature (London) 422, 147 (2003).

[6] N. K. Efremidis, S. Sears, D. N. Christodoulides, J. W. Fleischer, and M. Segev, Phys. Rev. E 66, 046602 (2002).

[7] D. Neshev, E. Ostrovskaya, Y. Kivshar, and W. Krolikowski, Opt. Lett. 28, 710 (2003).

[8] H. Martin, E. D. Eugenieva, Z. Chen, and D. N. Christodoulides, Phys. Rev. Lett. 92, 123902 (2004).

[9] T. Pertsch, U. Peschel, F. Lederer, J. Burghoff, M. Will, S. Nolte, and A. Tünnermann, Opt. Lett. 29, 468 (2004).

[10] U. Peschel, T. Persch, and F. Lederer, Opt. Lett. 23, 1701 (1998).

[11] T. Pertsch, P. Dannberg, W. Elflein, A. Bräuer, and F. Lederer, Phys. Rev. Lett. 83, 4752 (1999).

[12] R. Morandotti, U. Peschel, J. S. Aitchison, H. S. Eisenberg, and Y. Silberberg, Phys. Rev. Lett. 83, 4756 (1999).

[13] R. Iwanow, D. A. May-Arrioja, D. N. Christodoulides, G. I. Stegeman, Y. Min, and W. Sohler, Phys. Rev. Lett. 95, 053902 (2005).

[14] K. G. Makris, D. N. Christodoulides, O. Peleg, M. Segev, and D. Kip, Opt. Express 16, 10309 (2008).

[15] K. Shandarova, C. E. Rüter, D. Kip, K. G. Makris, D. N. Christodoulides, O. Peleg, and M. Segev, Phys. Rev. Lett. 102, 123905 (2009).

[16] S. Longhi, M. Marangoni, M. Lobino, R. Ramponi, P. Laporta, E. Cianci, and V. Foglietti, Phys. Rev. Lett. 96, 243901 (2006).
[17] I. L. Garanovich, A. A. Sukhorukov, and Y. S. Kivshar, Phys. Rev. E 74, 066609 (2006).

[18] R. Iyer, J. S. Aitchison, J. Wan, M. M. Dignam, and C. M. de Sterke, Opt. Express 15, 3212 (2007).

[19] A. Szameit, I. L. Garanovich, M. Heinrich, A. A. Sukhorukov, F. Dreisow, T. Pertsch, S. Nolte, A. Tünnermann, and Y. S. Kivshar, Nature Phys. 5, 271 (2009).

[20] Y. S. Kivshar and N. Flytzanis, Phys. Rev. A 46, 7972 (1992).

[21] A. A. Sukhorukov and Y. S. Kivshar, Opt. Lett. 27, 2112 (2002).

[22] K. Hizanidis, Y. Kominis, and N. K. Efremidis, Opt. Express 16, 18296 (2008).

[23] N. Malkova, I. Hromada, X. Wang, G. Bryant, and Z. Chen, Opt. Lett. 34, 1633 (2009).

[24] M. Heinrich, Y. V. Kartashov, L. P. R. Ramirez, A. Szameit, F. Dreisow, R. Keil, S. Nolte, A. Tünnermann, V. A. Vysloukh, and L. Torner, Opt. Lett. 34, 3701 (2009).

[25] P. Zhang, R. Egger, and Z. Chen, Opt. Express 17, 13151 (2009).

[26] N. K. Efremidis and K. Hizanidis, Opt. Express 13, 10571 (2005).

[27] A. Szameit, T. Pertsch, S. Nolte, A. Tünnermann, and F. Lederer, Phys. Rev. A 77, 043804 (2008).

[28] F. Fedele, J. Yang, and Z. Chen, Opt. Lett. 30, 1506 (2005).

[29] K. Staliunas and C. Masoller, Opt. Express 14, 10669 (2006).

[30] A. Szameit, Y. V. Kartashov, F. Dreisow, M. Heinrich, T. Pertsch, S. Nolte, A. Tünnermann, V. A. Vysloukh, F. Lederer, and L. Torner, Phys. Rev. Lett. 102, 153901 (2009).

[31] Y. V. Kartashov, A. Szameit, V. A. Vysloukh, and L. Torner, Opt. Lett. 34, 2906 (2009).

[32] F. Grossmann, T. Dittrich, P. Jung, and P. Hänggi, Phys. Rev. Lett. 67, 516 (1991).

[33] Y. S. Kivshar and D. K. Campbell, Phys. Rev. E 48, 3077 (1993). 